Article

\title{
Carapanolides $\mathbf{J}-\mathbf{L}$ from the Seeds of Carapa guianensis (Andiroba) and Their Effects on LPS-Activated NO Production
}

\author{
Yuuki Matsui ${ }^{1}$, Takashi Kikuchi ${ }^{1}$, Takanobu Inoue ${ }^{1}$, Osamu Muraoka ${ }^{2}$, Takeshi Yamada ${ }^{1}$ \\ and Reiko Tanaka ${ }^{1, *}$
}

1 Laboratory of Medicinal Chemistry, Osaka University of Pharmaceutical Sciences, 4-20-1 Nasahara, Takatsuki, Osaka 569-1094, Japan

2 Faculty of Pharmaceutical Sciences, Kinki University, 3-4-1 Kowakae, Higashiosaka, Osaka 577-8502, Japan

* Author to whom correspondence should be addressed; E-Mail: tanakar@gly.oups.ac.jp; Tel./Fax: +81-72-690-1084.

External Editor: Isabel C. F. R. Ferreira

Received: 5 September 2014; in revised form: 17 October 2014 / Accepted: 20 October 2014 / Published: 24 October 2014

\begin{abstract}
A novel gedunin and two novel phragmalin-type limonoids, named carapanolides J-L (compounds 1-3) as well as a known gedunin-type limonoid 4 were isolated from the seeds of Carapa guianensis (andiroba). Their structures were determined on the basis of $1 \mathrm{D}$ and 2D NMR spectroscopy and HRFABMS. Compounds 1-4 were evaluated for their effects on the production of NO in LPS-activated mouse peritoneal macrophages.
\end{abstract}

Keywords: Carapa guianensis; andiroba; seeds; gedunin; phragmalin; limonoids; NO production

\section{Introduction}

Carapa guianensis Aublet (Meliaceae), known locally as andiroba, is widely distributed in the Amazonas State of Brazil and its wood is extensively used as commercial timber [1]. Andiroba is a tall rainforest tree that grows up to $40 \mathrm{~m}$ in height. The indigenous people in the Amazon have used andiroba in many ways for centuries, and virtually all parts of the tree, as well as the seed oil, are utilized. It can be found growing wild throughout the Amazon rainforest, typically on rich soils, in swamps, and also in 
the alluvial flats, marshes, and uplands of the Amazon Basin. This tree can also be found wild or under cultivation in Brazil in the Islands region, Tocantins, Rio Solimoes, and near the seaside. It is one of the large-leafed trees of the rainforest and can be identified by its large and distinctively textured leaves. The andiroba tree produces a brown, woody, four-cornered nut with a diameter of 3-4 inches that resembles a chestnut. Andiroba oil is a rich source of essential fatty acids including oleic, palmitic, stearic, and linoleic acids. It yields up to $65 \%$ unsaturated fatty acids and can contain approximatoly $9 \%$ linoleic acid. Andiroba oil extracts yield up to $65 \%$ unsaturated fatty acids and can contain approximately 9\% linoleic acid. Extracts from its bark, flowers, and seeds have been used for centuries by the Amazonian people and exhibit various repellent [2], analgesic [3], anti-malarial [4], anti-inflammatory [5], anti-allergic [6], and antiplasmoidal [7] activities, as well as acute and subacute toxicities [8]. Our recent study on the components of the seed oil of Carapa guianasis revealed the structures of two new unusual 9,10-seco-mexicanolide-type limonoids, named carapanolides A and B [9], two novel carbon skeletal limonoids, named guianolides A and B [10], and carapanolides $\mathrm{C}-\mathrm{I}$ [11]. We herein describe the isolation and structural determination of three novel limonoids 1-3, named carapanolides J-L, and the effects of 1-3 and epoxyazadiradione (4) on the production of NO in LPS-activated mouse peritoneal macrophages. The structures of $\mathbf{1 - 3}$ were determined on the basis of NMR spectroscopy, including 1D and $2 \mathrm{D}\left({ }^{1} \mathrm{H},{ }^{1} \mathrm{H}-\mathrm{COSY}\right.$, NOESY, HSQC, HMBC) NMR, and FABMS.

\section{Results and Discussion}

The seed oil of Carapa guianensis $(2.03 \mathrm{~kg})$ was separated by silica gel column chromatography, medium-pressure liquid chromatography (MPLC), and reverse-phase HPLC to obtain three new limonoids 1-3 and a known limonoid 4, which was identified as epoxyazadiradione (Figure 1) [12].

The molecular formula of carapanolide $\mathbf{J}$ (1) was determined as $\mathrm{C}_{26} \mathrm{H}_{30} \mathrm{O}_{7}\left([\mathrm{M}+\mathrm{H}]^{+} m / z\right.$ 455.2075) based on HRFABMS. The IR and UV spectra showed bands assignable to a hydroxy group ( $v_{\max }$ $\left.3503 \mathrm{~cm}^{-1}\right)$, a six-membered ring ketone $\left(v_{\max } 1727 \mathrm{~cm}^{-1}\right)$, and an $\alpha, \beta$-unsaturated six-membered ring ketone $\left[v_{\max } 1671 \mathrm{~cm}^{-1} ; \lambda_{\max } 230 \mathrm{~nm}(\log \varepsilon 3.85)\right]$. The ${ }^{1} \mathrm{H}$ and ${ }^{13} \mathrm{C}$ NMR spectra (Table 1) exhibited signals assignable to five tertiary methyls $\left[\delta_{\mathrm{H}} 1.16,1.17,1.21,1.28\right.$, and 1.56]; two $\mathrm{CH}_{2}$ groups; five $s p^{3}$ methine groups, including three oxymethine $\left[\delta_{\mathrm{H}} 3.88(\mathrm{~s}), 4.47\right.$ (ddd), and 5.49 (s)]; five $s p^{3}$ quaternary carbons, including an oxycarbon $\left[\delta_{\mathrm{C}} 65.4(\mathrm{~s})\right]$; an $\alpha, \beta$-unsaturated six-membered ring ketone $\left[\delta_{\mathrm{H}} 5.84\right.$, $8.24($ each $1 \mathrm{H}, \mathrm{d}) ; \delta_{\mathrm{c}} 203.0(\mathrm{~s})$ ]; a saturated ketone [ $\delta_{\mathrm{C}} 207.7(\mathrm{~s})$ ]; $\delta$-lactone [ $\delta_{\mathrm{H}} 5.49(\mathrm{~s}) ; \delta_{\mathrm{C}} 166.4(\mathrm{~s})$ ]; and furan ring $\left[\delta_{\mathrm{H}} 6.39(\mathrm{dd}), 7.42(\mathrm{t}), 7.44(\mathrm{~m})\right]$. In the HMBC spectrum, long-range correlations were observed between Me-18 $\left(\delta_{\mathrm{H}} 1.21\right)$ and C-12, C-13, C-14 [ $\left.\delta_{\mathrm{C}} 65.4(\mathrm{~s})\right]$, and C-17 [ $\delta_{\mathrm{C}} 77.6(\mathrm{~d})$ ]; between Me-19 ( $\left.\delta_{\mathrm{H}} 1.56\right)$ and $\mathrm{C}-1$ ( $\left.\delta_{\mathrm{C}} 160.2\right), \mathrm{C}-5, \mathrm{C}-9$, and $\mathrm{C}-10$; between Me-28 $\left(\delta_{\mathrm{H}} 1.17\right)$ and $\mathrm{C}-3\left(\delta_{\mathrm{C}} 203.0\right)$, $\mathrm{C}-4, \mathrm{C}-5$, and C-29; between Me-29 ( $\left.\delta_{\mathrm{H}} 1.16\right)$ and C-3, C-4, C-5, and C-28; between Me-30 ( $\left.\delta_{\mathrm{H}} 1.28\right)$ and C-7 ( $\left.\delta_{\mathrm{C}} 207.7\right), \mathrm{C}-8$, C-9, and C-14; between H-11 ( $\left.\delta_{\mathrm{H}} 4.47\right)$ and C-8, C-9, C-10, C-12, and C-13; between H-15 $\left(\delta_{\mathrm{H}} 3.88\right)$ and $\mathrm{C}-8, \mathrm{C}-13, \mathrm{C}-14$ and $\mathrm{C}-16\left(\delta_{\mathrm{C}} 166.4\right)$; and between $\mathrm{H}-17\left(\delta_{\mathrm{H}} 5.49\right)$ and C-12, C-13, C-14, C-16, C-18, C-20 [ $\delta_{\mathrm{C}} 120.0$ (s)], C-21 [ $\delta_{\mathrm{C}} 141.1$ (d)], and C-22 [ $\delta_{\mathrm{C}} 109.7$ (s)] (Figure 2). An analysis of the ${ }^{1} \mathrm{H}-{ }^{1} \mathrm{H}$ COSY spectrum (H-1-H-2; H-5- $\mathrm{H}_{2}-6$; H-9-H-11- $\mathrm{H}_{2}-12$; and H-22-H-23) revealed the partial structure shown in Figure 2. The HMBC and ${ }^{1} \mathrm{H}-{ }^{1} \mathrm{H}$ COSY spectra revealed that 1 was a 11-hydroxy-7-deacetoxy-7-oxogedunin [13]. Selected NOESY correlations were shown in Figure 2. The secondary hydroxyl group at C-11 [ $\delta_{\mathrm{H}} 4.47$ (ddd)] was determined to have an $\alpha$ (equatorial) 
orientation because significant NOEs were observed between H-11 and Me-19, and Me-30, while

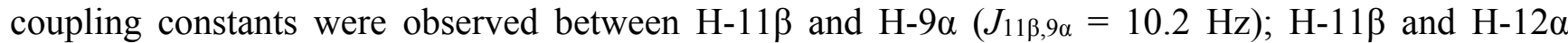

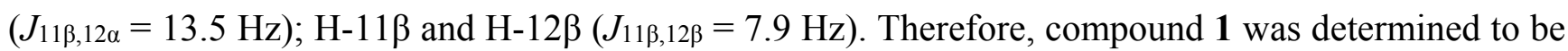
$11 \alpha$-hydroxy-7-deacetoxy-7-oxogedunin, which has been thus isolated in Nature for the first time.

Figure 1. Chemical structures for compounds $\mathbf{1}-\mathbf{4}$.

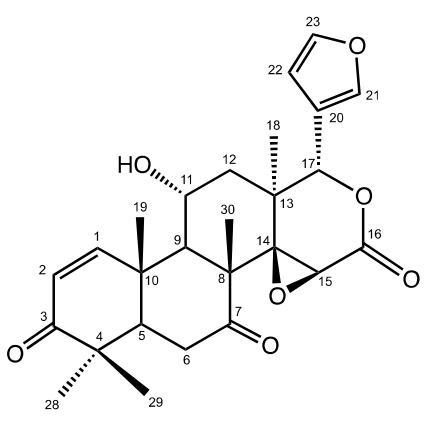

1

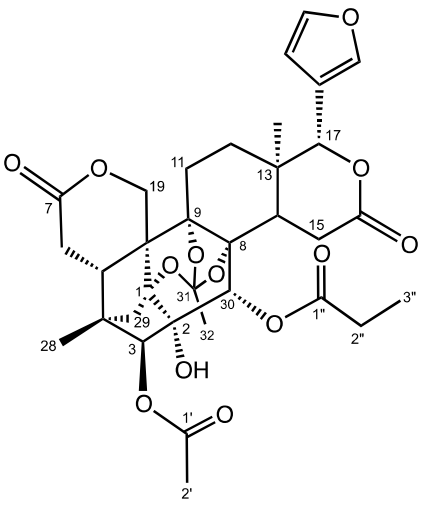

3

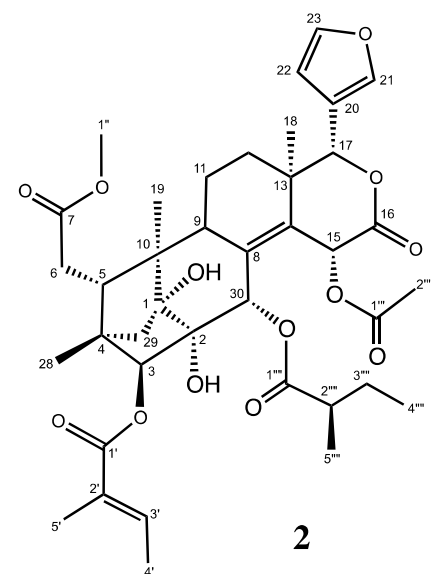<smiles>CC(=O)O[C@H]1CC2C(C)(C)C(=O)C=C[C@]2(C)C2CC[C@]3(C)[C@H](c4ccoc4)C(=O)[C@H]4O[C@]43[C@@]21C</smiles>

4

Figure 2. Key HMBC, COSY, and NOESY correlations for carapanolide J (1).
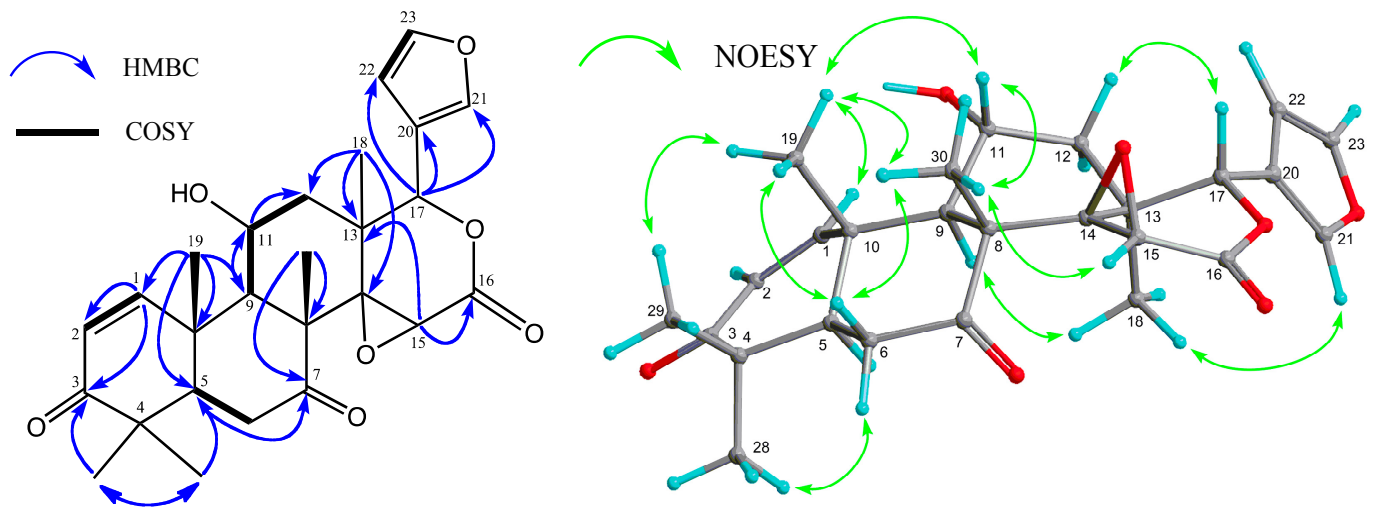
Table 1. ${ }^{1} \mathrm{H}(600 \mathrm{MHz})$ and ${ }^{13} \mathrm{C}(150 \mathrm{MHz})$ NMR spectroscopic data of compound $\mathbf{1 .}$

\begin{tabular}{|c|c|c|c|c|c|c|c|c|}
\hline \multirow{2}{*}{\multicolumn{2}{|c|}{ Position }} & \multicolumn{3}{|c|}{1} & \multirow{2}{*}{ Position } & \multicolumn{3}{|c|}{1} \\
\hline & & \multicolumn{2}{|r|}{${ }^{1} \mathbf{H}^{a}(J, \mathbf{H z})$} & ${ }^{13} \mathrm{C}^{b}$ & & \multicolumn{2}{|r|}{${ }^{1} \mathbf{H}^{a}(J, \mathbf{H z})$} & \multirow{2}{*}{$\begin{array}{l}{ }^{13} \mathbf{C}^{b} \\
65.4\end{array}$} \\
\hline 1 & & 8.24 & d $10.3(2)$ & 160.2 & 14 & & & \\
\hline 2 & & 5.84 & d 10.3 (1) & 124.9 & 15 & 3.88 & $\mathrm{~s}$ & 54.1 \\
\hline 3 & & & & 203.0 & 16 & & & 166.4 \\
\hline 4 & & & & 45.6 & 17 & 5.49 & $\mathrm{~s}$ & 77.6 \\
\hline 5 & & 2.21 & dd $3.2(6 \alpha), 14.6(6 \beta)$ & 53.9 & 18 & 1.21 & s & 20.6 \\
\hline \multirow[t]{2}{*}{6} & $\alpha$ & 2.38 & dd $3.2(5), 13.8(6 \beta)$ & 36.3 & 19 & 1.56 & $\mathrm{~s}$ & 20.9 \\
\hline & $\beta$ & 2.93 & dd $13.8(6 \alpha), 14.6(5)$ & & 20 & & & 120.0 \\
\hline 7 & & & & 207.7 & 21 & 7.44 & $\mathrm{~m}$ & 141.1 \\
\hline 8 & & & & 53.4 & 22 & 6.39 & dd $0.6(21), 1.7$ (23) & 109.7 \\
\hline 9 & & 2.45 & d $10.2(11)$ & 51.3 & 23 & 7.42 & t $1.7(21,22)$ & 143.3 \\
\hline 10 & & & & 40.9 & 28 & 1.17 & s & 20.7 \\
\hline 11 & $\beta$ & 4.47 & ddd $7.9(12 \beta), 10.2(9), 13.5(12 \alpha)$ & 67.3 & 29 & 1.16 & $\mathrm{~s}$ & 27.4 \\
\hline \multirow[t]{2}{*}{12} & $\alpha$ & 1.46 & dd $13.5(11), 13.8(12 \beta)$ & 44.6 & 30 & 1.28 & $\mathrm{~s}$ & 18.2 \\
\hline & $\beta$ & 2.21 & dd $7.9(11), 13.8(12 \alpha)$ & & $11-\mathrm{OH}$ & 1.83 & $\mathrm{~s}$ & \\
\hline 13 & & & & 38.0 & & & & \\
\hline
\end{tabular}

${ }^{a}$ Measured at $600 \mathrm{MHz}$ in $\mathrm{CDCl}_{3} ;{ }^{b}$ Measured at $150 \mathrm{MHz}$ in $\mathrm{CDCl}_{3}$. Assignments are based on HMBC spectrum.

Carapanolide K (2), which was isolated as a colorless amorphous solid, had the molecular formula $\mathrm{C}_{39} \mathrm{H}_{50} \mathrm{O}_{13}\left([\mathrm{M}+\mathrm{Na}]^{+} ; m / z\right.$ 749.3152, calcd. for 749.3155$)$ as determined by HRFABMS. The IR spectrum showed the presence of a hydroxyl at $v_{\max } 3446 \mathrm{~cm}^{-1}$, and ester groups at $v_{\max } 1766,1735$, and $1698 \mathrm{~cm}^{-1}$. The ${ }^{1} \mathrm{H}$-and ${ }^{13} \mathrm{C}$-NMR spectra (Table 2 ) indicated the presence of three methyls $\left[\delta_{\mathrm{H}} 0.83\right.$, $1.09,1.14($ each $3 \mathrm{H}, \mathrm{s})]$, an acetyl group $\left[\delta_{\mathrm{H}} 2.07(3 \mathrm{H}, \mathrm{s}), \delta_{\mathrm{C}} 21.1(\mathrm{q}), 169.9(\mathrm{~s})\right]$, 2-methylbutanoyl group $\left[\delta_{\mathrm{H}} 0.90(3 \mathrm{H}, \mathrm{t}), 1.13(3 \mathrm{H}, \mathrm{d}), 1.48\right.$ and $1.64($ each $\left.1 \mathrm{H}, \mathrm{m}), 2.38(1 \mathrm{H}, \mathrm{m}), \delta_{\mathrm{C}} 176.3(\mathrm{~s})\right]$, tigloyl group [ $\delta_{\mathrm{H}}$ $\left.1.77(3 \mathrm{H}, \mathrm{dd}), 1.98(3 \mathrm{H}, \mathrm{t}), 7.14(1 \mathrm{H}, \mathrm{qq}) ; \delta_{\mathrm{c}} 168.5(\mathrm{~s})\right]$, methoxycarbonyl group $\left[\delta_{\mathrm{H}} 3.72(3 \mathrm{H}, \mathrm{s}), \delta_{\mathrm{C}}\right.$ $52.0(\mathrm{q}), 174.2(\mathrm{~s})], \delta$-lactone $\left[\delta_{\mathrm{H}} 5.36(1 \mathrm{H}, \mathrm{s}), \delta_{\mathrm{C}} 80.3(\mathrm{~d}), 167.8(\mathrm{~s})\right]$, two tertiary hydroxyl groups $\left[\delta_{\mathrm{C}}\right.$ $77.0(\mathrm{~s}), 83.6(\mathrm{~s})]$, a tetrasubstituted double bond [ $\left.\delta_{\mathrm{C}} 134.9(\mathrm{~s}), 135.4(\mathrm{~s})\right]$, and furan ring [ $\delta_{\mathrm{H}} 6.47(\mathrm{dd})$, $7.41(\mathrm{t}), 7.58(\mathrm{t})]$. In the HMBC spectrum, cross-peaks were observed between Me-18 [ $\left.\delta_{\mathrm{H}} 1.09(\mathrm{~s})\right]$ and $\mathrm{C}-12, \mathrm{C}-13, \mathrm{C}-14$ [ $\delta_{\mathrm{C}} 135.4(\mathrm{~s})$ ], and C-17 [ $\delta_{\mathrm{C}} 80.3(\mathrm{~d})$ ]; between Me-19 [ $\left.\delta_{\mathrm{H}} 1.14(\mathrm{~s})\right]$ and C-1 [ $\delta_{\mathrm{C}} 83.6$ (s)], C-5, C-9, and C-10; between Me-28 [ $\left.\delta_{\mathrm{H}} 0.83(\mathrm{~s})\right]$ and C-3 [ $\left.\delta_{\mathrm{C}} 88.2(\mathrm{~d})\right], \mathrm{C}-4, \mathrm{C}-5$, and C-29; between H-3 [ $\left.\delta_{\mathrm{H}} 4.73(\mathrm{~s})\right]$ and C-1, C-2 [ $\left.\delta_{\mathrm{C}} 77.0(\mathrm{~d})\right], \mathrm{C}-4, \mathrm{C}-5, \mathrm{C}-28, \mathrm{C}-29, \mathrm{C}-30\left[\delta_{\mathrm{C}} 69.7\right.$ (d)], and

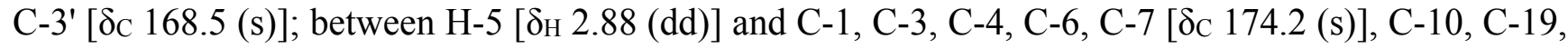
C-28, and C-29; between H-30 [ $\delta_{\mathrm{H}} 5.41$ (s)] and C-1, C-2, C-3, C-8 [ $\delta_{\mathrm{C}} 134.9(\mathrm{~s})$ ], C-9, and C-30' [ $\delta \mathrm{C}$ 176.3 (s)]. The positions of the hydroxyl, 2-methylbutanoyl, methoxycarbonyl, and tigloyl groups were identified by detailed ${ }^{1} \mathrm{H}-{ }^{1} \mathrm{H}$ COSY and $\mathrm{HMBC}$ correlations (Figure 3 ). In addition, the cross peaks between H-9 and H-30, and C-8 [ $\delta \mathrm{c} 134.9$ (s)]; between H-30, H-15, and C-14 [ $\delta_{\mathrm{c}} 135.4$ (s)] revealed that compound 2 was a phragmalin-8(14)-ene derivative [14]. In the NOESY spectrum, significant NOEs (Figure 3) were observed between $\mathrm{H}-3$ [ $\delta_{\mathrm{H}} 4.73$ (s)] and H-29 pro-S, H-30, and Me-28; between H-5 [ $\delta_{\mathrm{H}}$ 2.88 (dd)] and Me-28 and H-30; between Me-18 and H-11 $\alpha$ and H-12 $\alpha$; between Me-19 and H-11 $\alpha$, between $\mathrm{H}-15$ and H-17 $\beta, \mathrm{H}-30, \mathrm{H}-3$ ', H-5', and H-2"'; therefore, the 2-methylbutanoyl group at C-30 and acetoxy group at $\mathrm{C}-15$ were all $\alpha$ while the tigloyl group at $\mathrm{C}-3$ had a $\beta$ orientation. The configuration 
of the 2-methylbutanoyl group at C-30 was deduced to be $R$ because the chemical shift value and NOESY correlation were very similar to that of carapanolide $\mathrm{F}$ [11], which was determined as $2 R$ by single-crystal X-ray diffraction analysis.

Carapanolide L (3) was obtained as a colorless amorphous solid, and its molecular formula was established as $\mathrm{C}_{33} \mathrm{H}_{38} \mathrm{O}_{13}\left([\mathrm{M}+\mathrm{H}]^{+} ; m / z\right.$ 643.2391, calcd. for 643.2391) by HRFABMS, implying 15 degrees of unsaturation. The IR spectrum showed the presence of a hydroxyl at $v_{\max } 3352 \mathrm{~cm}^{-1}$, and ester groups at $v_{\max } 1742 \mathrm{~cm}^{-1}$. The ${ }^{1} \mathrm{H}$ - and ${ }^{13} \mathrm{C}$-NMR data indicated that eight of the 15 units of unsaturation came from two carbon-carbon double bonds and four ester carbonyls, including two lactone carbonyls. Therefore, the remaining degrees of unsaturation required 3 to be nonacyclic. The ${ }^{1} \mathrm{H}-$ and ${ }^{13} \mathrm{C}-\mathrm{NMR}$ spectra of $\mathbf{3}$ (Table 2) indicated the presence of two tertiary methyls [ $\delta_{\mathrm{H}} 1.00,1.13$ (each s)], an acetyl [ $\delta_{\mathrm{H}} 2.19(\mathrm{~s}) ; \delta_{\mathrm{C}} 21.6(\mathrm{q}), 170.4(\mathrm{~s})$ ], propanoyl [ $\delta_{\mathrm{H}} 1.09(3 \mathrm{H}, \mathrm{t}), 2.36(1 \mathrm{H}, \mathrm{dq}), 2.39(1 \mathrm{H}, \mathrm{dq})$; $\delta_{\mathrm{C}} 8.6(\mathrm{q}), 27.8(\mathrm{t}), 172.8(\mathrm{~s})$ ], and orthoacetyl group [ $\delta_{\mathrm{H}} 1.70(\mathrm{~s}) ; \delta_{\mathrm{C}} 21.0(\mathrm{q}), 119.6(\mathrm{~s})$ ], four methylenes, including an oxymethylene [ $\delta_{\mathrm{H}} 4.38$ and 4.77 (each $1 \mathrm{H}$, d), five $s p^{3}$ methines, including three oxymethines [ $\delta_{\mathrm{H}} 4.66(\mathrm{~s}), 5.35(\mathrm{~s})$, and $\left.5.71(\mathrm{~s})\right]$, a furan ring [ $\delta_{\mathrm{H}} 6.41(\mathrm{dd}), 7.44(\mathrm{t})$, and $7.48(\mathrm{~m})$ ], seven $s p^{3}$ quaternary carbons, including four oxycarbon $\left[\delta_{\mathrm{C}} 79.5(\mathrm{~s}), 85.4(\mathrm{~s}), 86.3(\mathrm{~s})\right.$, and $\left.86.4(\mathrm{~s})\right]$, two ester carbonyls [ $\delta_{\mathrm{C}} 170.4$, and 172.8 (each s)], and two lactone carbonyl [ $\delta_{\mathrm{C}} 169.8$, and $\left.171.1(\mathrm{~s})\right]$. An analysis of the ${ }^{1} \mathrm{H}-{ }^{1} \mathrm{H}$ COSY spectrum of 3 revealed the partial structures shown in bold face in Figure 4 . In the HMBC spectrum (Figure 4), cross-peaks were observed from Me-18 [ $\delta_{\mathrm{H}} 1.13$ (s)] to C-12, C-13, C-14, and $\mathrm{C}-17\left(\delta_{\mathrm{C}} 78.4\right)$; from Me-28 [ $\left.\delta_{\mathrm{H}} 1.00(\mathrm{~s})\right]$ to $\mathrm{C}-3, \mathrm{C}-4, \mathrm{C}-5$, and $\mathrm{C}-29$; from $\mathrm{H}-30\left[\delta_{\mathrm{H}} 5.71(\mathrm{~s})\right]$ to C-1 [ $\left.\delta_{\mathrm{C}} 85.4(\mathrm{~s})\right], \mathrm{C}-2$ [ $\left.\delta_{\mathrm{C}} 79.5(\mathrm{~s})\right], \mathrm{C}-3$ [ $\left.\delta_{\mathrm{C}} 83.9(\mathrm{~d})\right], \mathrm{C}-8$ [ $\delta_{\mathrm{C}} 86.4(\mathrm{~s})$ ], and C-9 [ $\left.\delta_{\mathrm{C}} 86.3(\mathrm{~s})\right]$ from H-14 $\left[\delta_{\mathrm{H}} 1.00(\mathrm{~s})\right]$ to C-8, C-9, C-12, C-13, C-15, and C-16 [ $\left.\delta_{\mathrm{C}} 169.8(\mathrm{~s})\right]$. Therefore, the planar structure of 3 was established as phragmalin-1,8,9-orthoacetate [13], and the positions of the hydroxyl, acetyl, and $n$-propyl groups were located at $\mathrm{C}-2, \mathrm{C}-3$, and $\mathrm{C}-30$ by detailed ${ }^{1} \mathrm{H}-{ }^{1} \mathrm{H}$ COSY and HMBC correlations (Figure 3). In the NOESY spectrum, significant NOEs (Figure 3) were observed between $\mathrm{H}-3$ [ $\delta_{\mathrm{H}} 4.73$

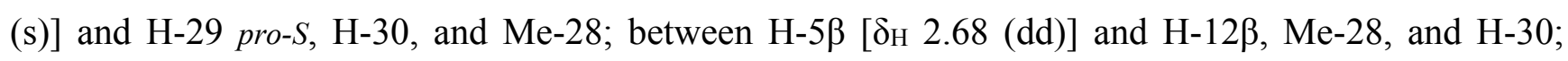
between $\mathrm{H}-15 \beta\left[\delta_{\mathrm{H}} 3.19(\mathrm{dd})\right]$ and $\mathrm{H}-30$; between $\mathrm{H}-17 \beta\left[\delta_{\mathrm{H}} 5.35(\mathrm{~s})\right]$ and $\mathrm{H}-12 \beta, \mathrm{H}-15 \beta, \mathrm{H}-22$, and $\mathrm{H}-30 \beta$, between Me-18 [ $\delta_{\mathrm{H}} 1.13$ (s)] and H-11 $\alpha, \mathrm{H}-12 \alpha$ and Me-32. Therefore, the relative structure of 3 was established as shown in Figure 1.

Figure 3. Selected ${ }^{1} \mathrm{H}-{ }^{1} \mathrm{H}$ COSY, HMBC and NOESY correlations for carapanolide K (2).
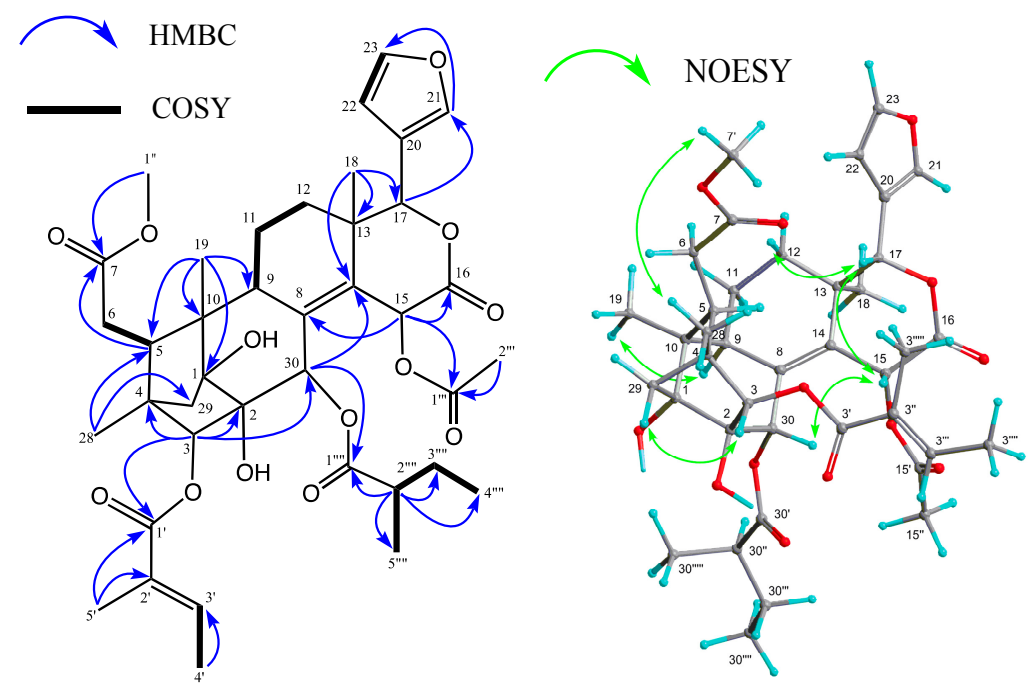
Table 2. ${ }^{1} \mathrm{H}-\mathrm{NMR}$ and ${ }^{13} \mathrm{C}-\mathrm{NMR}$ data for compounds 2 and $\mathbf{3 .}$

\begin{tabular}{|c|c|c|c|c|c|c|c|}
\hline \multirow{2}{*}{\multicolumn{2}{|c|}{ Position }} & \multicolumn{3}{|c|}{2} & \multicolumn{3}{|c|}{$\mathbf{3}$} \\
\hline & & & ${ }^{1} \mathbf{H}^{a}(J, \mathbf{H z})$ & ${ }^{13} \mathbf{C}^{b}$ & & ${ }^{1} \mathbf{H}^{a}(J, \mathbf{H z})$ & ${ }^{13} \mathrm{C}^{b}$ \\
\hline 1 & & & & 83.6 & & & 85.4 \\
\hline 2 & & & & 77.0 & & & 79.5 \\
\hline 3 & & 4.73 & $\mathrm{~s}$ & 88.2 & 4.66 & $\mathrm{~s}$ & 83.9 \\
\hline 4 & & & & 43.1 & & & 45.2 \\
\hline 5 & & 2.88 & dd $1.2(6 \mathrm{~B}), 5.3(6 \mathrm{~A})$ & 37.5 & 2.68 & dd 3.5 (6B), $5.5(6 \mathrm{~A})$ & 33.8 \\
\hline \multirow[t]{2}{*}{6} & A & 2.32 & d $5.3(5)$ & 33.7 & 2.46 & dd $5.5(5), 17.6(6 \mathrm{~B})$ & 31.0 \\
\hline & $\mathrm{B}$ & 2.33 & d $1.2(5)$ & & 2.66 & dd $3.5(5), 17.6(6 \mathrm{~A})$ & \\
\hline 7 & & & & 174.2 & & & 171.1 \\
\hline 8 & & & & 134.9 & & & 86.4 \\
\hline 9 & & 2.73 & d 7.7 & 35.9 & & & 86.3 \\
\hline 10 & & & & 47.5 & & & 44.7 \\
\hline \multirow[t]{2}{*}{11} & $\alpha$ & 1.70 & $\mathrm{~m}$ & 18.3 & 1.85 & dt $2.9(11 \alpha), 14.7(12 \alpha, \beta)$ & 25.7 \\
\hline & $\beta$ & 1.89 & $\mathrm{~m}$ & & 2.27 & $\mathrm{~m}$ & \\
\hline \multirow[t]{2}{*}{12} & $\alpha$ & 1.05 & $\mathrm{~m}$ & 28.5 & 1.48 & $\mathrm{~m}$ & 29.4 \\
\hline & $\beta$ & 1.40 & dt $3.2(12 \alpha), 14.1(11 \beta)$ & & 1.38 & $\mathrm{~m}$ & \\
\hline 13 & & & & 38.9 & & & 34.5 \\
\hline 14 & & & & 135.4 & 2.02 & dd $2.0(15 \beta), 10.5(15 \alpha)$ & 42.8 \\
\hline \multirow{2}{*}{15} & $\alpha$ & & & & 2.70 & dd $10.5(14), 20.0(15 \beta)$ & 26.4 \\
\hline & $\beta$ & 6.28 & d 2.4 & 64.2 & 3.19 & dd $2.0(14), 20.0(15 \alpha)$ & \\
\hline 16 & & & & 167.8 & & & 169.8 \\
\hline 17 & & 5.36 & $\mathrm{~s}$ & 80.3 & 5.35 & $\mathrm{~s}$ & 78.4 \\
\hline 18 & & 1.09 & $\mathrm{~s}$ & 16.7 & 1.13 & $\mathrm{~s}$ & 20.0 \\
\hline \multirow[t]{2}{*}{19} & $\alpha$ & 1.14 & $3 \mathrm{H}, \mathrm{s}$ & 17.3 & 4.77 & d $13.8(19 \beta)$ & 68.8 \\
\hline & $\beta$ & & & & 4.38 & d $13.8(19 \alpha)$ & \\
\hline 20 & & & & 120.5 & & & 120.8 \\
\hline 21 & & 7.58 & t $0.8(22)$ & 142.0 & 7.48 & t $0.8(22)$ & 140.8 \\
\hline 22 & & 6.47 & dd $0.8(21), 1.6(23)$ & 109.9 & 6.41 & dd $0.8(21), 1.8(23)$ & 109.6 \\
\hline 23 & & 7.41 & t $1.6(22)$ & 143.0 & 7.44 & t $1.8(22)$ & 143.4 \\
\hline 28 & & 0.83 & $\mathrm{~s}$ & 14.8 & 1.00 & $\mathrm{~s}$ & 13.6 \\
\hline \multirow{2}{*}{29} & pro- $R$ & 1.58 & d $11.0(29$ pro-S $)$ & 39.8 & 1.80 & d $11.1(29$ pro-S $)$ & 38.3 \\
\hline & pro-S & 1.86 & d $11.0(29$ pro- $R)$ & & 2.25 & d 11.1 (29 pro- $R)$ & \\
\hline 30 & & 5.41 & $\mathrm{~s}$ & 69.7 & 5.71 & $\mathrm{~s}$ & 70.0 \\
\hline 31 & & & & & & & 119.6 \\
\hline 32 & & & & & 1.70 & $\mathrm{~s}$ & 21.0 \\
\hline $1^{\prime}$ & & & & 168.5 & & & 170.4 \\
\hline $2^{\prime}$ & & & & 130.0 & 2.19 & $\mathrm{~s}$ & 21.6 \\
\hline $3^{\prime}$ & & 7.14 & qq $7.0\left(4^{\prime}\right), 1.1\left(5^{\prime}\right)$ & 12.2 & & & \\
\hline $4^{\prime}$ & & 1.77 & dd $1.1\left(5^{\prime}\right), 7.0\left(3^{\prime}\right)$ & 139.2 & & & \\
\hline $5^{\prime}$ & & 1.98 & t $1.1\left(3^{\prime}, 4^{\prime}\right)$ & 14.5 & & & \\
\hline $1 "$ & & 3.72 & $\mathrm{~s}$ & 52.0 & & & 172.8 \\
\hline \multirow[t]{2}{*}{$2^{\prime \prime}$} & A & & & & 2.36 & dq 7.5 (3"), 9.7 (2"B) & 27.8 \\
\hline & B & & & & 2.39 & dq $7.5(3 "), 9.7(2 " \mathrm{~A})$ & \\
\hline 3" & & & & & 1.09 & $3 \mathrm{H}, \mathrm{t} 7.5(2 \mathrm{~A}$, 2 "B) & 8.6 \\
\hline $1 " '$ & & & & 169.9 & & & \\
\hline 2"' & & 2.07 & $\mathrm{~s}$ & 21.1 & & & \\
\hline $1 " '$ & & & & 176.3 & & & \\
\hline \multirow[t]{2}{*}{$2^{\prime \prime \prime \prime}$} & A & 2.38 & $\mathrm{~m}$ & 40.9 & & & \\
\hline & $\mathrm{B}$ & & & & & & \\
\hline \multirow[t]{2}{*}{ 3"'" } & A & 1.48 & $\mathrm{~m}$ & 26.5 & & & \\
\hline & B & 1.64 & $\mathrm{~m}$ & & & & \\
\hline 4"'" & & 0.90 & t 7.3 (3"'"A, 3"'"B) & 16.4 & & & \\
\hline $5^{\prime \prime \prime \prime}$ & & 1.13 & d $7.0\left(2^{\prime \prime \prime \prime}\right)$ & 11.3 & & & \\
\hline
\end{tabular}

${ }^{a}$ Measured at $600 \mathrm{MHz}$ in $\mathrm{CDCl}_{3} ;{ }^{b}$ Measured at $150 \mathrm{MHz}$ in $\mathrm{CDCl}_{3}$. Assignments are based on HMBC spectrum. 
Figure 4. Key HMBC, ${ }^{1} \mathrm{H}-{ }^{1} \mathrm{H}$ COSY, and NOESY correlations of carapanolide L (3).
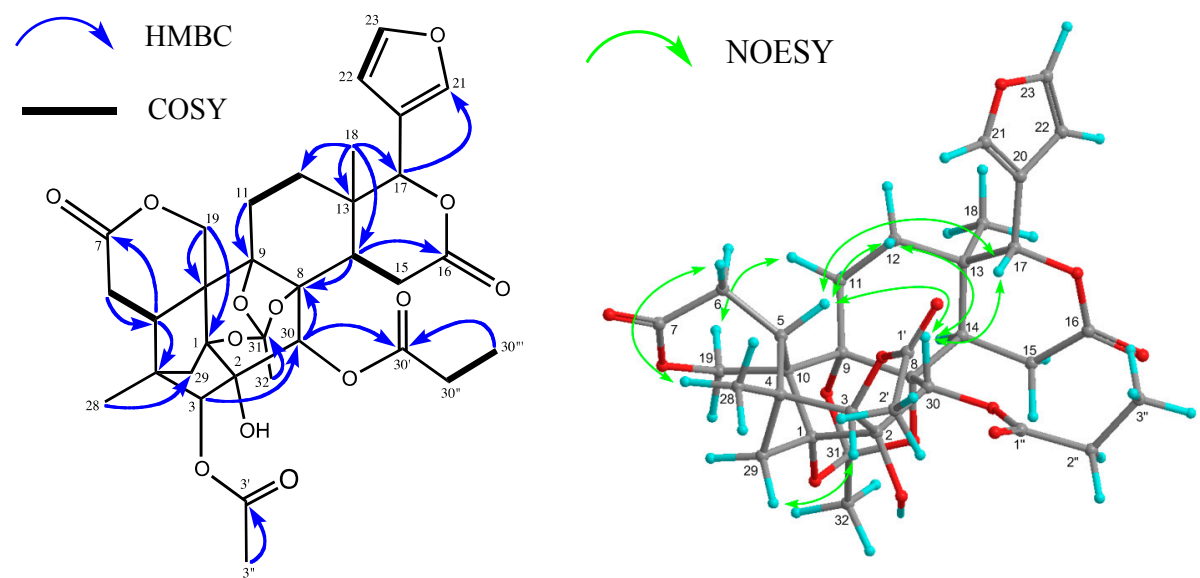

Physiological nitric oxide (NO) is involved in blood pressure regulation and blood flow distribution, whereas its overexpression may induce tissue injury, multiple organ dysfunction, and death, as well as systemic inflammatory responses in sepsis, such as hypotension, cardiodepression, and vascular hyporeactivity [15]. In the present study, four limonoids and L-NMMA, an inducible nitric oxide synthase (iNOS) inhibitor, were evaluated for their inhibitory effects on NO production in LPS-stimulated RAW264.7 cells (Table 3). To determine safe concentrations, the cytotoxicities of these limonoids against RAW 264.7 were assessed by the MTT assay. Compounds $\mathbf{1}$ and $\mathbf{3}$ showed non-toxicities at 3-100 $\mu \mathrm{M}$, whereas 4 and 2 exhibited moderate cytotoxicities (IC50 4: $21.3 \mu \mathrm{M} ; \mathbf{2}$ : $15.2 \mu \mathrm{M})$. In the inhibitory assay of NO production, compound 1 showed similar inhibitory activities (produced NO $83.4 \%$ at $10 \mu \mathrm{M} ; 61.8 \%$ at $30 \mu \mathrm{M} ; 16.8 \%$ at $100 \mu \mathrm{M}$ ) to the positive control, L-NMMA (produced NO $79.3 \%$ at $10 \mu \mathrm{M} ; 58.2 \%$ at $30 \mu \mathrm{M} ; 39.9 \%$ at $100 \mu \mathrm{M}$ ), with no cytotoxicities. Compound 4 exhibited superior inhibitory activities on NO production at non-toxic concentrations (produced NO $74.0 \%$ at $3 \mu \mathrm{M} ; 30.0 \%$ at $10 \mu \mathrm{M}$ ) to those of L-NMMA. These results suggested that compound 1 may be valuable as potential inhibitors of NO production.

Table 3. Inhobitory effects of NO production by limonoids from the seeds of Carapa guianensis.

\begin{tabular}{|c|c|c|c|c|c|c|}
\hline \multirow{2}{*}{ Compound } & & \multicolumn{4}{|c|}{ Concentration $(\mu \mathrm{M})$} & \multirow[b]{2}{*}{$\mathrm{IC}_{50}(\boldsymbol{\mu} \mathrm{M}$} \\
\hline & & 3 & 10 & 30 & 100 & \\
\hline \multirow[t]{2}{*}{1} & Produced NO $(\%)^{a}$ & $92.1 \pm 1.5$ & $83.4 \pm 3.1$ & $61.8 \pm 1.8$ & $16.8 \pm 0.0$ & 37.4 \\
\hline & Cell viability (\%) ${ }^{a}$ & $102.4 \pm 0.8$ & $101.0 \pm 1.7$ & $102.8 \pm 0.6$ & $103.4 \pm 1.8$ & $>100$ \\
\hline \multirow[t]{2}{*}{2} & Produced NO (\%) & $78.6 \pm 1.9$ & $58.3 \pm 2.8$ & $25.8 \pm 7.0$ & $7.1 \pm 1.2$ & 12.0 \\
\hline & Cell viability (\%) & $81.4 \pm 0.8$ & $65.6 \pm 0.2$ & $33.6 \pm 6.3$ & $0.4 \pm 0.4$ & 15.2 \\
\hline \multirow[t]{2}{*}{3} & Produced NO (\%) & $95.6 \pm 2.5$ & $95.4 \pm 1.2$ & $95.4 \pm 2.9$ & $78.4 \pm 2.3$ & $>100$ \\
\hline & Cell viability (\%) & $97.6 \pm 0.6$ & $97.3 \pm 1.3$ & $100.5 \pm 0.4$ & $94.4 \pm 1.0$ & $>100$ \\
\hline \multirow[t]{2}{*}{4} & Produced NO (\%) & $74.0 \pm 5.0$ & $30.0 \pm 2.3$ & $7.5 \pm 1.0$ & $3.9 \pm 1.8$ & 5.9 \\
\hline & Cell viability (\%) & $93.6 \pm 1.4$ & $99.7 \pm 0.8$ & $6.8 \pm 0.3$ & $3.3 \pm 0.3$ & 21.3 \\
\hline \multirow[t]{2}{*}{ L-NMMA $^{b}$} & Produced NO (\%) & $93.0 \pm 3.3$ & $79.3 \pm 0.8$ & $58.2 \pm 2.4$ & $39.9 \pm 1.7$ & 53.7 \\
\hline & Cell viability (\%) & $103.5 \pm 0.5$ & $102.0 \pm 1.5$ & $94.1 \pm 1.4$ & $96.5 \pm 2.5$ & $>100$ \\
\hline
\end{tabular}

${ }^{a}$ Produced NO (\%) and cell viability (\%) were determined based on the absorbance at $570 \mathrm{~nm}$, respectively, by comparison with values for DMSO $(100 \%)$. Each value represents the mean \pm standard error (S.E.) of three determinations. The concentration of DMSO in the sample solution was $2 \mu \mathrm{L} / \mathrm{mL}$; ${ }^{b}$ Positive control. 


\section{Experimental Section}

\subsection{General Procedures}

Melting points were determined on a Yanagimoto micro-melting point apparatus and were uncorrected. Optical rotations were measured using a JASCO DIP-1000 digital polarimeter. IR spectra were recorded using a Perkin-Elmer $1720 \mathrm{X}$ FTIR spectrophotometer. ${ }^{1} \mathrm{H}$ - and ${ }^{13} \mathrm{C}-\mathrm{NMR}$ spectra were obtained on an Agilent vnmrs 600 spectrometer with standard pulse sequences, operating at 600 and $150 \mathrm{MHz}$, respectively. $\mathrm{CDCl}_{3}$ was used as the solvent and TMS, as the internal standard. FABMS were recorded on a JEOL-7000 mass spectrometer. Column chromatography was carried out over silica gel (70-230 mesh, Merck, Darmstadt, Germany) and MPLC was carried out with silica gel (230-400 mesh, Merck). HPLC was run on a JASCO PU-1586 instrument equipped with a differential refractometer (RI 1531). Fractions obtained from column chromatography were monitored by TLC (silica gel $60 \mathrm{~F}_{254}$, Merck).

\subsection{Plant Material}

The oil of (2.03 kg) Carapa guianensis AUBLET (Meliaceae) was collected in the Amazon, Brazil, in March 2013. Kindly provided by Mr. Akira Yoshino (who is a representative of the NGO "Green Heart Love Amazon Project"). A voucher specimen (CGS-01-2) was deposited in the Herbarium of the Laboratory of Medicinal Chemistry, Osaka University of Pharmaceutical Sciences.

\subsection{Isolation of Compounds 1-4}

The seed oil of Carapa guianensis AUBLET (Meliaceae) $(2.03 \mathrm{~kg})$ was dissolved in $\mathrm{CHCl}_{3}(1 \mathrm{~L})$ and the $\mathrm{CHCl}_{3}$ solution was subjected to CC (silica gel $14 \mathrm{~kg}$ ), to afford seven fractions: Fraction A (Fr. No. $1-85,1.512 \mathrm{~kg}$ ) was eluted with $n$-hexane- $\mathrm{CHCl}_{3}=1: 1$, B (Fr. No. 86-179, $229.1 \mathrm{~g}$ ) was eluted with $\mathrm{CHCl}_{3}$, C (Fr. No. 180-220, $29.3 \mathrm{~g}$ ) was eluted with $\mathrm{CHCl}_{3}$-EtOAc = 5:1, D (Fr. No. 221-225, $13.2 \mathrm{~g}$ ) was eluted with $\mathrm{CHCl}_{3}$-EtOAc = 2:1, E (Fr. No. 226-265, 84.5 g) was eluted with $\mathrm{CHCl}_{3}$-EtOAc = 2:1, F (Fr. No. 266-290, 25.3 g) was eluted with EtOAc, G (Fr. No. 291-315, 72.8 g) was eluted with EtOAc: $\mathrm{MeOH}=5: 1$, and $\mathrm{H}$ (Fr. No. 316-333, 45.4 g) was eluted with MeOH. Residue D was rechromatographed over a silica gel column (CC) (230-400 mesh, $300 \mathrm{~g})$ eluted with $n$-hexane- EtOAc (1:1) to give 13 fractions: D1 (Fr. No. 1-35, 1.52 g), D2 (Fr. No. 36-49, 0.81 g), D(3) (Fr. No. 50-88, 0.70 g), D(4) (Fr. No. 89-115, 0.53 g), D(5) (Fr. No. 116-130, 0.60 g), D(6) (Fr. No. 131-140, 0.52 g), D(7) (Fr. No. 141-205, 0.47 g), D(8) (Fr. No. 206-215, 0.51 g), D(9) (Fr. No. 216-220, 0.42 g), D(10) (Fr. No. 221-240, 0.40 g), D(11) (Fr. No. 241-250, 1.11 g), and D(12) (Fr. No. 251-313, 1.36 g). Fraction D(6) was subjected to CC (230-400 mesh, $40 \mathrm{~g})$ eluted with $n$-hexane-EtOAc $(3: 1)$ to give an amorphous solid $\left(24.1 \mathrm{mg}\right.$ ) that was separated by HPLC (ODS, $75 \% \mathrm{MeOH}$, at $25{ }^{\circ} \mathrm{C}$, flow rate $4.0 \mathrm{~mL} \cdot \mathrm{min}^{-1}, \mathrm{UV}=220 \mathrm{~nm}$, column $250 \times 20 \mathrm{~mm}$ i.d., $\left.5 \mu \mathrm{m}\right)$ to give compounds $2(6.2 \mathrm{mg})$ and 3 (1.79 mg). Fraction D(8) was subjected to CC (230-400 mesh, $40 \mathrm{~g})$ eluted with $n$-hexane-EtOAc (3:1) to give an amorphous solid (34.0 mg) that was subjected to CC (230-400 mesh, $40 \mathrm{~g})$ eluted with $n$-hexane-EtOAc (3:1) to give an amorphous solid that was purified by HPLC (ODS, 75\% MeOH, at $25^{\circ} \mathrm{C}$, flow rate $4.0 \mathrm{~mL} \cdot \mathrm{min}^{-1}, \mathrm{UV}=220 \mathrm{~nm}$, column $250 \times 20 \mathrm{~mm}$ i.d., $5 \mu \mathrm{m}$ ) to give compounds 1 $(7.5 \mathrm{mg})$ and $4(3.8 \mathrm{mg})$. Fraction $\mathrm{D}(9)$ was subjected to CC (230-400 mesh, $30 \mathrm{~g})$ eluted with 
$n$-hexane-EtOAc (3:1) to give an amorphous solid $(25.5 \mathrm{mg}$ ) that was separated by HPLC (ODS, 70\% $\mathrm{MeOH}$, at $25{ }^{\circ} \mathrm{C}$, flow rate $4.0 \mathrm{~mL} \cdot \mathrm{min}^{-1}, \mathrm{UV}=220 \mathrm{~nm}$, column $250 \times 20 \mathrm{~mm}$ i.d., $5 \mu \mathrm{m}$ ) to give compound $3(6.2 \mathrm{mg})$.

\subsection{Analytical Data}

Compound 1. Colorless crystals; mp $172-174{ }^{\circ} \mathrm{C}$ (from $\left.\mathrm{MeOH}-\mathrm{CHCl}_{3}\right) ;[\alpha]_{\mathrm{D}}^{26}-18.7^{\circ}\left(c \mathrm{c} .1, \mathrm{CHCl}_{3}\right.$ ); HRFABMS $m / z: 455.2075[\mathrm{M}+\mathrm{H}]^{+}\left(\mathrm{C}_{26} \mathrm{H}_{31} \mathrm{O}_{7}\right.$, calcd for 455.2080); UV (EtOH) $\lambda_{\max } \mathrm{nm}(\log \varepsilon): 230$ (3.85), 237 (3.80), 248 (3.63); IR (KBr) $v_{\max } \mathrm{cm}^{-1}$; $3503(\mathrm{OH}), 2926,1727(\mathrm{O}-\mathrm{C}=\mathrm{O}), 1671(\mathrm{C}=\mathrm{C}-\mathrm{C}=\mathrm{O})$; ${ }^{1} \mathrm{H}-$ and ${ }^{13} \mathrm{C}-\mathrm{NMR}$, see Table 1. FABMS $m / z$ (rel. int.): $477\left([\mathrm{M}+\mathrm{Na}]^{+}, 15\right), 455\left([\mathrm{M}+\mathrm{H}]^{+}, 71\right), 83(100)$.

Compound 2. Colorless amorphous solids; $[\alpha]_{\mathrm{D}}^{26}-72.2^{\circ}\left(c \quad 0.1, \mathrm{CHCl}_{3}\right)$; HRFABMS $m / z: 749.3152$ $[\mathrm{M}+\mathrm{Na}]^{+}\left(\mathrm{C}_{39} \mathrm{H}_{50} \mathrm{O}_{13} \mathrm{Na}\right.$, calcd for 749.3155); UV $\lambda_{\max }(\mathrm{EtOH}) \mathrm{nm}(\log \varepsilon): 227$ (4.19), 304 (3.98), 315 (4.00), 334 (3.72); IR (KBr) $v_{\max } \mathrm{cm}^{-1}: 3446(\mathrm{OH}), 2967,1766$ and 1735, 1698; ${ }^{1} \mathrm{H}-$ and ${ }^{13} \mathrm{C}-\mathrm{NMR}$, see Table 2. FABMS $m / z$ (rel. int.): 749 (33) $\left([\mathrm{M}+\mathrm{Na}]^{+}, 3\right), 727\left([\mathrm{M}+\mathrm{H}]^{+}, 100\right)$.

Compound 3. Colorless amorphous solids; $[\alpha]_{\mathrm{D}}^{26}-46.8^{\circ}\left(c \quad 0.1, \mathrm{CHCl}_{3}\right)$; HRFABMS $m / z$ : 643.2391 $[\mathrm{M}+\mathrm{H}]^{+}\left(\mathrm{C}_{33} \mathrm{H}_{38} \mathrm{O}_{13}\right.$, calcd for 643.2391); UV $\lambda$ max $(\mathrm{EtOH}) \mathrm{nm}(\log \varepsilon): 208$ (1.26), IR (KBr) $v_{\max } \mathrm{cm}^{-1}$ : $3352(\mathrm{OH}), 1742(\mathrm{O}-\mathrm{C}=\mathrm{O}) ;{ }^{1} \mathrm{H}-$ and ${ }^{13} \mathrm{C}-\mathrm{NMR}$, see Table 2. FABMS $m / z$ (rel. int.): 665 (33) $\left([\mathrm{M}+\mathrm{Na}]^{+}\right.$, 12), $643\left([\mathrm{M}+\mathrm{H}]^{+}, 100\right)$.

\subsection{Determination of RAW264.7 Cell Proliferation}

RAW264.7 cell proliferation was examined according to a method reported previously [16] with some modifications. Briefly, RAW264.7 cells $\left(5 \times 10^{4}\right.$ cells in $\left.100 \mu \mathrm{L}\right)$ were seeded onto 96-well microplates, and incubated for $24 \mathrm{~h}$. D-MEM $(100 \mu \mathrm{L})$ containing test samples (final concentration of 100, 30, 10, or $3 \mu \mathrm{M}$ ) dissolved in DMSO (final concentration 0.2\%) was added. After the cells had been treated for $24 \mathrm{~h}$, the MTT solution was added. After $3 \mathrm{~h}$ of incubation, 20\% sodium dodecyl sulfate (SDS) in $0.1 \mathrm{M} \mathrm{HCl}$ was added to dissolve the formazan produced by the cells. The absorbance of each well was read at $570 \mathrm{~nm}$ using a microplate reader. The optical density of vehicle control cells was assumed to be $100 \%$.

\subsection{Inhibitory Assay of NO Production}

An inhibitory assay of nitric oxide production was performed according to a method reported previously [17] with slight modifications. Briefly, RAW264.7 cells $\left(5 \times 10^{4}\right.$ cells in $\left.100 \mu \mathrm{L}\right)$ were seeded onto 96-well microplates, and incubated for $24 \mathrm{~h}$. D-MEM $(100 \mu \mathrm{L})$ containing test samples (final concentration of $100,30,10$, or $3 \mu \mathrm{M}$ ) dissolved in DMSO (final concentration $0.2 \%$ ) and LPS (final concentration of $5 \mu \mathrm{g} / \mathrm{mL}$ ) were added. After cells had been treated for $24 \mathrm{~h}, 50 \mu \mathrm{L}$ of $0.1 \%$ $N$-(1-naphtyl)ethylenediamine in $\mathrm{H}_{2} \mathrm{O}$ and $50 \mu \mathrm{L}$ of $1 \%$ sulfanylamide in $5 \%$ phosphoric acid were added. After being incubated for $30 \mathrm{~min}$, the absorbance of each well was read at $570 \mathrm{~nm}$ using a microplate reader. The optical density of vehicle control cells was assumed to be $100 \%$. 


\section{Conclusions}

A novel gedunin and two novel phragmalin-type limonoids, named carapanolides J-L (compounds 1-3), as well as a known gedunin-type limonoid $\mathbf{4}$ were isolated from the seeds of Carapa guianensis (andiroba). Their structures were determined by spectroscopic analyses. Compound 1 showed similar inhibitory activities (produced NO $83.4 \%$ at $10 \mu \mathrm{M} ; 61.8 \%$ at $30 \mu \mathrm{M} ; 16.8 \%$ at $100 \mu \mathrm{M}$ ) to positive control, L-NMMA (produced NO 79.3\% at $10 \mu \mathrm{M} ; 58.2 \%$ at $30 \mu \mathrm{M} ; 39.9 \%$ at $100 \mu \mathrm{M}$ ), with no cytotoxicity. Known compound $\mathbf{4}$ exhibited superior inhibitory NO production activities at non-toxic concentrations (produced NO $74.0 \%$ at $3 \mu \mathrm{M} ; 30.0 \%$ at $10 \mu \mathrm{M}$ ) to those of L-NMMA. These results suggest that compound $\mathbf{1}$ may be a valuable potential inhibitor of NO production.

\section{Acknowledgments}

We thank Katsuhiko Minoura and Mihoyo Fujitake (this university) for the NMR and MS measurements.

\section{Author Contributions}

Y. Matsui and T. Inoue performed the isolation and structure elucidation. T. Kikuchi contributed to evaluation of bioactivities. T. Yamada, O. Muraoka and R. Tanaka prepared the manuscript and supervised whole research project.

\section{Conflicts of Interest}

The authors declare no conflict of interest.

\section{References}

1. Tan, Q.G.; Luo, X.D. Meliaceous limonoids: chemistry and biological activities. Chem. Rev. 2011, 111, 7437-7522.

2. Prophiro, J.S.; da Silva Mario, A.N.; Kanis, L.A.; da Rocha, L.C.B.P.; Duque-Luna, J.E.; da Silva, O.S. First report on susceptibility of wild Aedes aegypty (Diptera: Culicidae) using Carapa guianensis (Meliaceae) and Copaifera sp. (Leguminosae). Parasitol. Res. 2012, 110, 7699-7705.

3. Penido, C.; Costa, K.A.; Pennaforte, R.J.; Costa, M.F.S.; Pereira, J.F.G.; Siani, A.C.; Henriques, M.G.M.O. Anti-allergic effects of natural tetranortriterpenoids isolated from Carapa guianensis Aublet on allergen-induced vascular permeability and hyperalgesia. Inflamm. Res. 2005, 54, 295-303.

4. Bickii, J.; Njifutie, N.; Foyere, J.A.; Basco, L.K.; Ringwald, P.J. In vitro antimalarial activity of limonoids from Khaya grandifoliola C.D.C. (Meliaceae). J. Ethnopharmacol. 2000, 69, 27-33.

5. Penido, C.; Conte, F.P.; Chagas, M.S.S.; Rodrigue, C.A.B.; Pereira, J.F.G.; Henriques, M.G.M.O. Antiinflammatory effects of natural tetranortriterpenoids isolated from Carapa guianensis Aublet on zymosan-induced arthritis in mice. Inflamm. Res. 2006, 55, 457-464. 
6. Ferraris Fausto, K.; Rodrigues, R.; da Silva, V.P.; Figueiredo, R.; Penido, C.; Henriques, M.G.M.O. Modulation of $\mathrm{T}$ lymphocyte and eosinophil functions in vitro by natural tetranortriterpenoids isolated from Carapa guianensis Aublet. Int. Immunopharmacol. 2011, 11, 1-11.

7. Miranda Junior, R.N.C.; Dolabela, M.F.; da Silva, M.N.; Povoa, M.M.; Maia, J.G.S. Antiplasmoidal activity of the andiroba (Carapa guianensis Aublet., Meliaceae) oil and its limonoid-rich fraction. J. Ethnopharmacol. 2012, 142, 679-683.

8. Costa-Silva, H.; Lima, C.R.; Silva, E.J.R.; Araujo, A.V.; Fraga, M.C.C.R.; Ribeiro, E.; Ribwiro, A.; Arruda, A.C.; Lafayette, S.S.L.; Wanderley, J. Acute and subacute toxicity of the Carapa guianensis Aublet (Meliaceae) seed oil. J. Ethnopharmacol. 2008, 116, 495-500.

9. Inoue, T.; Nagai, Y.; Mitooka, A.; Ujike, R.; Muraoka, O.; Yamada, T.; Tanaka, R. Carapanolides $\mathrm{A}$ and $\mathrm{B}$ : unusual 9,10-seco-mexicanolides having a $2 R, 9 S$-oxygen bridge from the seeds of Carapa guianensis. Tetrahedron Lett. 2012, 53, 6685-6688.

10. Inoue, T.; Matsui, Y.; Kikuchi, T.; In, Y.; Yamada, T.; Muraoka, O.; Matsunaga, S.; Tanaka, R. Guianolides A and B, New Carbon Skeletal Limonoids from the seeds of Carapa guianensis. Org. Lett. 2013, 15, 3018-3021.

11. Inoue, T.; Matsui, Y.; Kikuchi, T.; In, Y.; Muraoka, O.; Yamada, T.; Tanaka, R. Carapanolides C-I from the seeds of andiroba (Carapa guianensis, Meliaceae). Fitoterapia 2014, 96, 56-64.

12. Malathi, R.; Rajan, S.S.; Mohan Kumar, R.; Narasimhan, S.; Ravikumar, K. Epoxyazadiradione. Acta Crystallogr. 2007, E63, 2483-2485.

13. Tanaka, Y.; Yamada, T.; In, Y.; Muraoka, O.; Kajimito, T.; Tanaka, R. Absolute stereostructure of Andirolides A-G from the flower of Carapa guianensis (Meliaceae). Tetrahedron 2011, 67, 782-792.

14. Ravangpai, W.; Sommit, D.; Teerawatananond, T.; Sinpranee, N.; Palaga, T.; Pengpreecha, S.; Muangsin, N.; Pudhom, K. Limonoids from seeds of Thai Xylocarpus moluccensis. Bioorg. Med. Chem. Lett. 2011, 21, 4485-4489.

15. Kirkeboen, K.A.; Strand, O.A. The role of nitric oxide in sepsis-an overview. Acta Anaesthesiol. Scand. 1999, 43, 275-288.

16. Yamada, T.; Muroga, Y.; Jinno, M.; Kajimoto, T.; Usami, Y.; Numata, A.; Tanaka, R. New class azaphilone produced by a marine fish-derived Chaetomium globosum. The stereochemistry and biological activities. Bioorg. Med. Chem. 2011, 19, 4106-4113.

17. Yamasaki, F.; Machida, S.; Nakata, A.; Nugroho, A.E.; Hirasawa, Y.; Kaneda, T.; Morita, H. Haworforbins A-C, new phenolics from Haworthia cymbiformis. J. Nat. Med. 2013, 67, 212-216.

Sample Availability: Not available.

(C) 2014 by the authors; licensee MDPI, Basel, Switzerland. This article is an open access article distributed under the terms and conditions of the Creative Commons Attribution License (http://creativecommons.org/licenses/by/4.0/). 